\title{
Método otimizado de avaliação de processos
}

\author{
Valquíria M. Fagundes, Sílvia R. Lopes, Raquel M. P. Gutierrez \\ Fundação CPqD Centro de Pesquisa e Desenvolvimento em Telecomunicações - CPqD, \\ Campinas-SP, Brasil \\ \{fagundes, slopes, moreda\}@ cpqd.com.br
}

\begin{abstract}
Resumo. Este artigo descreve a experiência da organização brasileira $C P q D$ (Fundação CPqD Centro de Pesquisa e Desenvolvimento em Telecomunicações) no desenvolvimento de um método para execução de avaliações integradas de um sistema de gestão, aderente a várias normas e modelos. O CPqD necessitava de um processo de avaliação menos oneroso para a verificação de seus processos e com isto buscou desenvolver um método otimizado de avaliação.
\end{abstract}

\begin{abstract}
This article describes the experience of CPqD (Fundação CPqD Centro de Pesquisa e Desenvolvimento em Telecomunicações), a Brazilian organization, in the development of a method for performing integrated assessments of a management system, which is compliant to several standards and models. CPqD needed a less expensive evaluation process for the verification of their processes and thus sought to develop an optimized method of evaluation.
\end{abstract}

\section{Introdução}

O CPqD é uma organização focada na inovação, com base em tecnologias da informação e comunicação (TICs), e tem o objetivo de contribuir para a competitividade e a inclusão digital do País. Cultiva valores como inovação, excelência, respeito, objetividade, confiança e parceria na sua atuação em pesquisa (por meio de seu Programa de Pesquisa e Desenvolvimento), no mercado e na criação de novas empresas, disseminando assim suas tecnologias de sistemas de software, tecnologias de produtos e serviços tecnológicos.

O Sistema de Gestão do $\mathrm{CPqD}$ abrange processos para o desenvolvimento de software e de produtos, serviços de laboratório, serviços de calibração, serviços de consultoria, certificação de produtos, entre outros. É certificado e atende às normas ABNT NBR ISO 9001:2008 [ABNT 2008], ABNT NBR ISO 14001:2004 [ABNT 2004], ABNT NBR ISO/IEC ISO 17025:2005 [ABNT 2005] e ao nível de maturidade 3 do modelo CMMI for Development v. 1.3 (CMMI-DEV) [CMMI 2010]. Para que essas certificações fossem mantidas e a organização buscasse a melhoria contínua de seus processos, estabeleceu-se em seu processo de garantia da qualidade a realização de avaliações periódicas internas.

Até 2009 a empresa utilizava um método de avaliação de processos baseado nos métodos de avaliação tradicionais e realizava avaliações específicas para verificar o atendimento a cada uma das normas ou modelos aplicáveis na organização, utilizando a norma ABNT NBR ISO 19011:2012 [ABNT 2012], para auditorias internas dos processos frente às normas ISO 9001 e 14001, e realizando avaliações mensais para 
verificação dos processos em todos os projetos de desenvolvimento de software, garantindo o atendimento à prática de PPQA (Process and Product Quality Assurance) do CMMI-DEV. Havia em torno de 21 profissionais envolvidos com as avaliações de processo, sendo 7 deles profissionais envolvidos na verificação de aderência dos processos às normas ISO (136 instâncias de processos verificadas trimestralmente, intercalando-se avaliações internas e externas) e 12 profissionais envolvidos na verificação de aderência dos processos ao modelo CMMI-DEV (560 instâncias de processos verificadas mensalmente).

Em 2009, diante das implicações da grande crise de 2008-2009 [Wessel 2010], a direção do $\mathrm{CPqD}$, atenta à mitigação de potenciais riscos, determinou um "período de austeridade" - um período no qual todas as áreas da organização foram motivadas a sugerir melhorias para a redução de custos e o aumento de produtividade, sem entretanto abrir mão da qualidade de seus produtos e de seus processos. Essa diretriz levou as áreas a reverem seus processos e a sua forma de operação, buscando simplificação, otimização, maior produtividade e ganho de qualidade.

Após as avaliações que mantiveram as certificações das normas ISO 9001:2008 e ISO 14001:2004 e do modelo CMMI-DEV, ocorridas durante o ano de 2009, e que atestaram o elevado grau de maturidade do Sistema de Gestão da organização, a unidade administrativa de Assessoria da Qualidade da organização (AQ), responsável pela avaliação dos processos e pela manutenção do Sistema de Gestão, não só atuou na melhoria de processos produtivos como também promoveu a melhoria em seus próprios processos e procedimentos operacionais. Nesse contexto, um dos desafios a serem superados era melhorar a produtividade das avaliações, procurando gastar menos recursos e gerar menores impactos nas áreas avaliadas, a partir de um método de avaliação inovador que pudesse atender tanto às normas ISO como ao modelo CMMI$\mathrm{DEV}$. O método desenvolvido, que hoje está em uso pelo $\mathrm{CPqD}$, é descrito neste artigo.

\section{Buscando um novo método de avaliação}

$\mathrm{O}$ método de avaliação de processos utilizado pelo $\mathrm{CPqD}$ exigia muito esforço para a geração dos resultados esperados, sendo que nem sempre a organização conseguia obter uma visão sistêmica dos problemas do seu Sistema de Gestão. Tendo como foco a necessidade de melhorar a produtividade nas avaliações internas de processo, a AQ procurou elaborar um método de avaliação que permitisse integrar as avaliações específicas dos processos do Sistema de Gestão e que:

a) atendesse ao mesmo tempo aos requisitos da norma ABNT NBR ISO 19011:2012 e à prática de PPQA do CMMI-DEV;

b) possibilitasse uma visão sistêmica dos problemas na execução de seus processos e fornecesse insumos para melhoria contínua dos processos;

c) garantisse que a maioria das instâncias de processos em execução na organização pudessem ser verificadas, preservando uma relação custo-benefício adequada.

Para definição inicial do novo método de avaliação, foi aplicado o PDCA, iniciando com a pesquisa e leitura de artigos e bibliografia relacionada [Shrum, Chrissis \& Konrad 2004], [Góes 2010], [Schaaf 2008], histórico das avaliações anteriores, benchmarking com outras empresas, experiência de auditores ISO e CMMI-DEV. Após 
a primeira avaliação utilizando o método, foram coletadas as lições aprendidas para ajustes e nova aplicação.

O método definido procurou respeitar as características próprias das normas e dos modelos aplicáveis na organização, o que permitiu eliminar as sobreposições, atenuar as deficiências e manter as individualidades, executar a avaliação de forma integrada e obter maior produtividade e objetividade. Além disto, ao estudar a definição do método, observou-se uma oportunidade de inovação com relação à aderência do método à prática de PPQA do CMMI-DEV. Observou-se que os casos mais típicos de implementação dessa prática preveem que todos os projetos de software passem por avaliação em todos os processos em fase de execução. O método desenvolvido prevê que apenas uma amostra de projetos da organização passe por avaliação completa e então, a partir dos problemas identificados nessa amostra, os demais projetos da organização são verificados com foco apenas na identificação da repetitividade desses problemas.

\section{Descrição do método de avaliação}

Como todos os processos do Sistema de Gestão do CPqD estão aderentes às normas e aos modelos aplicáveis, foi decidido que as avaliações seriam realizadas utilizando-se os processos como guia de verificação, tornando mais padronizada a forma de avaliar.

O método desenvolvido prevê inicialmente avaliações trimestrais completas, podendo essa periodicidade ser ajustada em função do histórico e da análise das avaliações realizadas. Em cada avaliação completa são verificados: um conjunto de projetos de desenvolvimento de software, por meio da avaliação de todas as instâncias dos processos em execução, e um conjunto de processos de áreas da organização, abrangendo áreas como compras, laboratórios, consultoria, serviços de calibração, entre outras.

A Figura 1 ilustra as principais etapas do método de avaliação do CPqD. Em seguida, essas etapas são descritas.

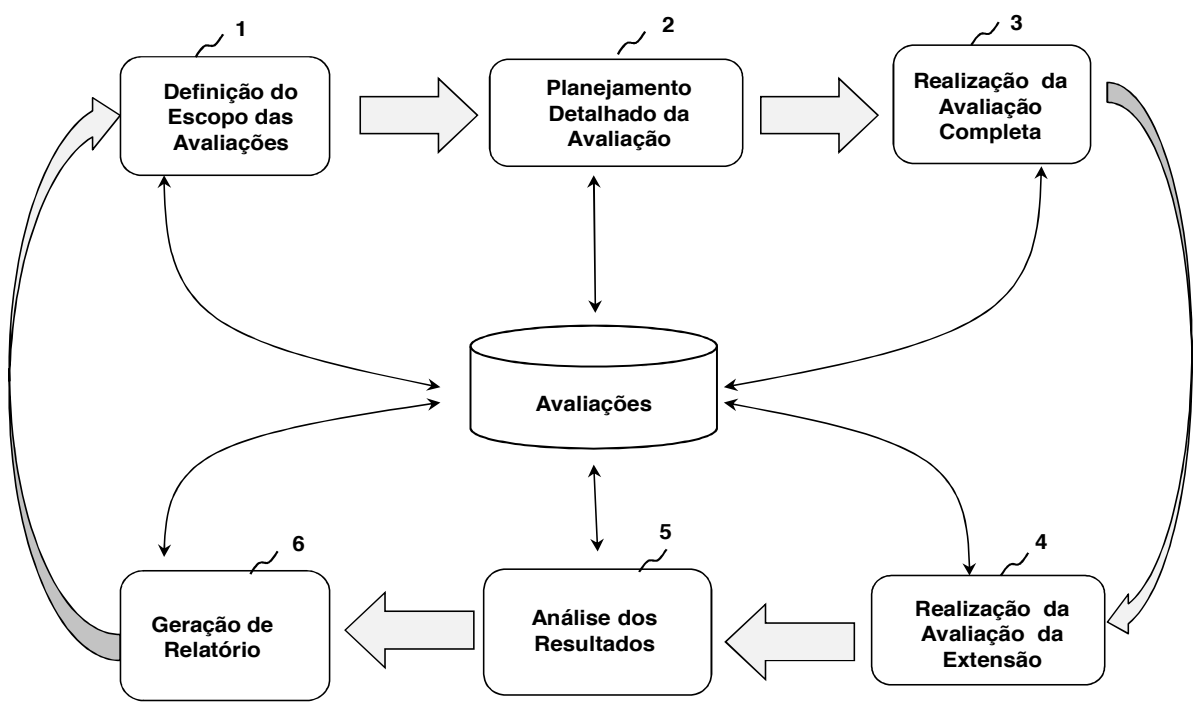

Figura 1. Etapas da avaliação 


\subsection{Definição do Escopo das Avaliações (1)}

No início do ano são verificados os processos que cada área do CPqD executa, com base no tipo de produto produzido na área, e é realizada a Definição do Escopo das Avaliações (1). Nessa etapa, é elaborado um macroplanejamento, no qual se define em qual avaliação do ano cada processo e área serão verificados, procurando-se balancear o número de processos e áreas em cada avaliação, de modo que ao final do ano todos os processos de todas as áreas tenham sido verificados. O histórico de avaliações anteriores passadas é verificado analisando-se os principais problemas encontrados, de modo que um processo de determinada área possa ser verificado mais de uma vez ao ano (caso a área tenha apresentado muitos problemas, por exemplo). Para as áreas que possuem projetos de desenvolvimento de software, os projetos a serem verificados são selecionados dentro do planejamento detalhado de cada avaliação (2). A Tabela 1 mostra um exemplo de um planejamento macro.

Tabela 1. Macroplanejamento da avaliação

\begin{tabular}{|c|c|c|c|c|c|}
\hline \multicolumn{6}{|c|}{ Programa de Avaliações Internas Integradas } \\
\hline & & \multicolumn{4}{|c|}{2011} \\
\hline & & Avaliação 1 & Avaliação 2 & Avaliação 3 & Avaliação 4 \\
\hline \multirow[t]{2}{*}{$\begin{array}{c}\text { Código } \\
\text { Área }\end{array}$} & Processo/Aspecto por Área & $\begin{array}{c}21 / \operatorname{mar} / 11 \\
a \\
25 / m a r / 11 \\
\end{array}$ & $\begin{array}{c}13 / j u n / 11 \\
a \\
17 / j u n / 11 \\
\end{array}$ & $\begin{array}{c}\text { 19/set/11 } \\
\text { a } \\
\text { 23/set/11 }\end{array}$ & $\begin{array}{c}\text { 28/nov/11 } \\
\text { a } \\
02 / \text { dez11 }\end{array}$ \\
\hline & Gestão e Suporte Empresarial & & & & \\
\hline \multirow[t]{2}{*}{1000} & Alta Direção & $\mathrm{x}$ & & $\mathrm{x}$ & \\
\hline & Consultoria & & & & \\
\hline \multirow{2}{*}{$\begin{array}{l}\mathbf{2 1 0 0} \\
2101 \\
\end{array}$} & Diretoria 1 & & & & \\
\hline & Gerência de Segurança da Informação & $\mathrm{x}$ & & $\mathrm{x}$ & \\
\hline \multirow{2}{*}{$\begin{array}{l}2102 \\
2300\end{array}$} & Gerência de Estratégia e Planejamento de Serviços & & $\mathrm{x}$ & & $\mathrm{x}$ \\
\hline & Diretoria 2 & & & & \\
\hline \multirow[t]{2}{*}{2301} & Gerência de Escritório de Projetos & $\mathrm{x}$ & & $\mathrm{x}$ & $\mathrm{x}$ \\
\hline & Serviços Laboratoriais & & & & \\
\hline \multirow[t]{2}{*}{2400} & Diretoria 3 & & & & \\
\hline & Laboratorio 1 & $\mathrm{x}$ & & $\mathrm{x}$ & \\
\hline
\end{tabular}

\subsection{Planejamento Detalhado da Avaliação (2)}

Perto da data planejada para realização da avaliação é realizado o Planejamento Detalhado da Avaliação (2), no qual são definidos os processos e as áreas a serem avaliados. No caso das áreas com projetos de desenvolvimento de software, são selecionados os projetos a serem avaliados. No planejamento detalhado também são definidos:

a) o ambiente de avaliação onde os avaliadores armazenarão as informações relacionadas à execução. $\mathrm{O} \mathrm{CPqD}$ utiliza uma ferramenta que auxilia na execução da avaliação, com um checklist dos itens a serem verificados em cada processo;

b) os avaliadores que verificarão cada área e processo, com o objetivo de alocar um determinado processo para apenas um avaliador. Procura-se alocar um especialista no assunto e os avaliadores são todos treinados no método de avaliação, nas normas e nos modelos aplicáveis à organização. Nesse tipo de abordagem, ocorre um aumento na produtividade, na uniformidade e na profundidade da avaliação, já que o avaliador alocado tem experiência na avaliação do processo, e também ocorre um aumento na probabilidade de se detectar os problemas existentes;

c) os projetos de desenvolvimento de software que serão verificados durante a Realização da Avaliação Completa (3) e aqueles que serão analisados 
apenas durante a Realização da Avaliação da Extensão (4). A diferença é que, na avaliação completa, todos os processos dos projetos selecionados são verificados e, na avaliação de extensão, os demais projetos são verificados apenas com foco na repetitividade do conjunto de problemas identificados na avaliação completa (essa questão é mais bem descrita no item 3.4).

Para a seleção dos projetos que participarão da avaliação completa, utiliza-se um método formal de decisão, por meio do qual se verifica o total dos projetos existentes em cada área. São estabelecidos critérios objetivos para seleção dos projetos e um ranking de relevância de cada critério, atribuindo-se um peso. Atribui-se então uma nota para cada projeto em cada critério. Em seguida, calcula-se a nota média ponderada de cada projeto:

$$
\mathrm{M}=\Sigma_{\mathrm{i}=1}^{\mathrm{n}} \quad\left(\mathrm{N}_{(\mathrm{i})} * \mathrm{P}_{(\mathrm{i})}\right) / \Sigma_{\mathrm{i}=1}^{\mathrm{n}} \quad \mathrm{P}_{(\mathrm{i})}
$$

onde: $M=$ nota média de cada projeto; $N(i)=$ nota de cada projeto para cada critério "i" e $P(i)=$ peso de cada critério "i".

São selecionados os projetos de maior nota de cada área selecionada, procurandose obter uma boa amostra do número total de projetos.

Para definição dos critérios usados na seleção dos projetos, foi realizado um estudo dos dados históricos das avaliações anteriores, e foi possível identificar um padrão de comportamento na execução dos processos. Isso levou à definição de critérios que possibilitassem selecionar projetos com maior possibilidade de apresentar desvios de processo ao longo de sua execução. Por exemplo: um projeto de desenvolvimento de um produto novo, com duração pequena e grande esforço de execução, tem mais chances de desvio do que um projeto de produto estável, com duração longa e esforço médio.

Na Tabela 2 são apresentados os critérios utilizados pelo CPqD e na Tabela 3, um exemplo usado para a seleção dos projetos utilizando esses critérios.

Tabela 2. Critérios para seleção de projetos

\begin{tabular}{|c|c|}
\hline Tabela - Critérios & \\
\hline Critérios & Nota para o critério \\
\hline \% Conclusão & $\begin{array}{l}\text { Acima de } 60 \% . \text { Nota }=10 \\
30,1 \%-60 \% . \text { Nota }=5 \\
\text { Até } 30 \% . \text { Nota }=1\end{array}$ \\
\hline $\begin{array}{l}\text { Maturidade/ estabilidade } \\
\text { do produto }\end{array}$ & $\begin{array}{l}\text { Estável. Nota }=1 \\
\text { Médio. Nota }=5 \\
\text { Novo. Nota }=10\end{array}$ \\
\hline Complexidade & $\begin{array}{l}\text { Análise do esforço e duração: } \\
\text { Esforço: } \\
\text { Até } 1500 \mathrm{~h} \text {. Nota = E1 } \\
1501 \mathrm{~h} \text { a } 3500 \mathrm{~h} \text {. Nota = E5 } \\
\text { Acima de } 3500 \mathrm{~h} \text {. Nota =E10 } \\
\text { Duração: } \\
\text { De } 50 \text { a } 100 \text { dias. Nota = D10 } \\
101 \text { a } 200 \text { dias. Nota = D5 } \\
\text { Acima de } 200 \text { dias. Nota = D1 } \\
\text { Esforço } x \text { Duração: } \\
\text { E10 e D5. Nota }=10 \\
\text { E10 e D10. Nota }=10 \\
\text { E5 e D10. Nota }=10 \\
\text { E1 e D10. Nota }=5 \\
\text { E1 e D5. Nota }=5 \\
\text { E5 e D5. Nota }=5 \\
\text { E10 e D1. Nota }=1 \\
\text { E5 e D1. Nota }=1 \\
\text { E1 e D1. Nota }=1\end{array}$ \\
\hline
\end{tabular}

Tabela 3. Tabela para seleção de projetos 


\begin{tabular}{|c|c|c|c|c|}
\hline \multirow{4}{*}{ Projetos } & \multicolumn{3}{|c|}{ Critérios } & \multirow{4}{*}{ Resultado } \\
\hline & \% Conclusão & $\begin{array}{c}\text { Maturidade/ } \\
\text { estabilidade } \\
\text { do produto }\end{array}$ & Complexidade & \\
\hline & \multicolumn{3}{|c|}{ Pesos } & \\
\hline & 1 & 5 & 10 & \\
\hline \multicolumn{5}{|l|}{ Diretoria 1} \\
\hline PROJETO FLORESTA & 1 & 5 & 1 & 2,25 \\
\hline PROJETO ÁRVORE & 5 & 1 & 10 & 6,88 \\
\hline PROJETO LAGOA & 1 & 5 & 5 & 4,75 \\
\hline \multicolumn{5}{|l|}{ Diretoria 2} \\
\hline PROJETO RAKUNA & 1 & 5 & 10 & 7,88 \\
\hline PROJETO SAVANA & 10 & 10 & 1 & 4,38 \\
\hline
\end{tabular}

\subsection{Realização da Avaliação Completa (3)}

Após a etapa de Planejamento Detalhado da Avaliação, inicia-se a Realização da Avaliação Completa (3), na qual todos os processos das áreas e projetos selecionados são verificados.

O avaliador verifica os processos utilizando o checklist disponível na ferramenta de avaliação, anota as evidências do que foi avaliado e identifica oportunidades de melhoria, melhores práticas e problemas encontrados.

Durante a avaliação, a troca de informações entre os avaliadores é estabelecida para que um possível problema identificado em um processo tenha o seu impacto verificado em outros processos (cross-match).

Ao final dessa avaliação, os avaliadores participam de uma consolidação dos resultados, garantindo que todos estão de acordo com o que foi identificado e nenhum ponto foi esquecido. Os problemas identificados e consolidados são validados com as áreas e os projetos em que foram encontrados.

Com relação à verificação dos projetos de desenvolvimento de software, é possível afirmar que, por conta da amostra de projetos selecionados (de acordo com os critérios) e da profundidade aplicada, grande parte dos principais problemas da organização são identificados. Vale lembrar que os critérios foram definidos nas etapas anteriores, com o intuito de selecionar projetos cuja aplicação do processo tivesse probabilidade de apresentar maiores desvios.

\subsection{Realização da Avaliação da Extensão (4)}

Após a Realização da Avaliação Completa (3), inicia-se a etapa de Realização da Avaliação da Extensão (4) nos demais projetos de software. Também é possível selecionar uma amostra dos projetos que passarão pela avaliação de extensão, de forma similar ao que é feito nos projetos para avaliação completa. Porém, deve-se garantir que nenhum projeto da organização seja concluído sem ter passado por um dos dois tipos de avaliação.

A avaliação de extensão é realizada da seguinte forma: com base no conjunto de problemas identificados na avaliação completa e armazenados na ferramenta de avaliação, verifica-se a ocorrência de repetição de cada um desses problemas nos demais projetos selecionados para extensão. 
Pela aplicação da extensão, é possível avaliar os demais processos dos projetos em execução, de maneira mais racional, ágil e eficiente, sem perder a qualidade do resultado desejado.

Ao final dessa avaliação, obtém-se um outro conjunto de problemas e oportunidades de melhoria identificados nos projetos, que são consolidados e armazenados na ferramenta de avaliação.

\subsection{Análise dos Resultados (5)}

Os resultados obtidos na avaliação completa e na de extensão são analisados. São gerados então gráficos que demonstram, por exemplo, como se encontra a execução dos processos do Sistema de Gestão da organização, enfatizando-se de maneira clara onde estão os processos que mais apresentam problemas e requerem uma atenção especial. $\mathrm{Na}$ análise também é possível identificar quais são os problemas pontuais e os sistêmicos, facilitando a definição do foco na tomada de ações de correção e melhoria.

São realizadas análises de tendência dos processos, com o objetivo de diminuir os riscos para a organização e definir ações preventivas, sendo essas análises realizadas levando-se em consideração o histórico de avaliações anteriores.

Por meio da análise de repetibilidade dos problemas, são identificadas causasraiz, visando a eliminação do problema e a melhoria dos processos.

\subsection{Geração de Relatório (6)}

Ao final da aplicação do método, é gerado um relatório contendo as análises realizadas e seus resultados, que servirá de histórico para novos ciclos de avaliação.

Esse relatório é disponibilizado e compartilhado nos diversos níveis da organização, servindo como insumo para a tomada de decisão no que diz respeito a investimentos em projetos de melhoria.

\section{Resultados obtidos}

Com a aplicação do método de avaliação desenvolvido, foram obtidos os seguintes resultados:

a) o método possibilitou minimizar o impacto na rotina de trabalho das áreas avaliadas, melhorou a produtividade e a objetividade na realização desse tipo de serviço, possibilitou melhor visualização dos problemas do sistema de gestão da organização e reduziu em $15 \%$ o esforço para esse tipo de atividade;

b) foi também possibilitada a redução no número necessário de profissionais para realização das avaliações, sendo que hoje há 11 profissionais envolvidos com as avaliações e a manutenção do Sistema de Gestão do $\mathrm{CPqD}$;

c) houve a solicitação de um pedido de patente para o método desenvolvido, pois a abordagem definida com relação à avaliação de projetos de desenvolvimento de software é inovadora não é usual e está aderente à prática de PPQA do CMMI-DEV; 
d) foi comprovada a aderência do método ao CMMI-DEV e às normas ISO aplicáveis no CPqD, por meio da manutenção das certificações em 2012.

\section{Conclusão}

O método desenvolvido pode ser aplicado em qualquer empresa cujo sistema de gestão possui processos relativamente estáveis e bem institucionalizados, o que beneficiará tais empresas, que, por sua vez, poderão obter maior produtividade e objetividade na avaliação de seu sistema de gestão.

O CPqD aplica o método de avaliação descrito neste artigo há 3 anos - desde 2010. O método proporcionou maior objetividade nas avaliações e auxiliou o CPqD a manter o foco em suas ações de melhoria de governança, aumentando a eficiência do investimento feito para garantir processos e produtos de melhor qualidade.

\section{Referências}

ABNT - Associação Brasileira de Normas Técnicas. Sistemas da qualidade: Requisitos: ISO 9001:2008.

ABNT - Associação Brasileira de Normas Técnicas. Sistemas da qualidade: Requisitos: ISO 14001:2004.

ABNT - Associação Brasileira de Normas Técnicas. Sistemas da qualidade: Requisitos: ISO/IEC ISO 17025:2005.

ABNT - Associação Brasileira de Normas Técnicas. Sistemas da qualidade: Requisitos: ISO/IEC ISO 19011:2012.

CMU/SEI-2010-TR-033, CMMI for Development, Version 1.3.

Góes, S. (2010) “Ação Corretiva e Ação Preventiva White Paper". Disponível em: $<$ http://executivebc.com.br/arquivos_pdf/pdf505.pdf $>$.

Schaaf, K. (2008) “Planning a Combined CMMI and a AS91000 Appraisal”. Disponível em: $\quad<$ http://fleet.management6.com/Planning-a-Combined-CMMI-and-AS9100Appraisal-download-w8011.pdf $>$.

Shrum, S., Chrissis, M. and Konrad M. (2004) CMMI - Guidelines for Process Integration and Product Improvement, Addison Wesley.

Wessel, D. (2010) "Did 'Great Recession' Live Up to the Name?", In: The Wall Street Journal. 